Submitted to the 1988 IEEE AP-S International Symposium in Syracuse, New York, June 1988

\title{
Diode-Grid Oscillators
}

\author{
ZORANA B. POPOVIĆ and DAVID B. RUTLEDGE
}

Division of Engineering and Applied Sciences

California Institute of Technology

Pasadena, CA 91125

\begin{abstract}
Loading a two-dimensional grid with active devices offers a means of combining the power of solid-state devices in the microwave and millimeter-wave range. The grid structure allows a large number of negative resistance devices to be combined. This approach is attractive because the active devices do not need an external locking signal, and the combining is done in free space. The loaded grid is a simple planar structure suitable for monolithic integration. Preliminary measurements on a 9-diode grid at $10 \mathrm{GHz}$ show power-combining and frequencylocking without an external locking signal.
\end{abstract}

\section{INTRODUCTION}

Gunn diode and IMPATT oscillators in the microwave and millimeter-wave range are reliable and light in weight, but have limited RF powers (typically a few watts at $10 \mathrm{GHz}$ to a few hundred milliwatts at $100 \mathrm{GHz}$ ). Recently, there has been much interest in developing quantum-well oscillators at frequencies above $100 \mathrm{GHz}$, but the output powers are quite low. Kesan et al. [1] predict the output power to be $10 \mu \mathrm{W}$ at $400 \mathrm{GHz}$. Since radar and imaging array applications require high power levels, coherent powercombining of a large number of solid-state devices becomes attractive. Mink $[\mathbf{2}]$ gave a theoretical analysis of quasi-optical combining, and Stephan [3] and Dinger et al. [4] demonstrated free-space combining and inter-injection locking of two and three devices. We propose a grid loaded with Gunn-diodes, which allows the power of a large number of devices to be combined. The idea of using a diode-grid structure for phase-shifting and frequency multiplication was investigated by Lam et al. [5] and Jou et al. [6]. Lam fabricated and characterized a grid with 1600 Schottky diodes. He showed that such a grid can be accurately modeled as a component in a simple transmission-line equivalent circuit, and that the grid has an inductance which can be found from statics.

\section{DIODE-GRID OSCILLATORS}

The proposed Gunn-diode grid is shown on Fig. 1(a). The RF currents are induced in the conductive lines parallel to the electric field. The perpendicular lines do not perturb the RF fields, and they are used for DC bias lines. The spacing between the diodes is small $\left(\lambda_{0} / 6\right)$, so the diode-grid can be treated as a uniform active sheet. The




structure should have a high reliability, since a few defective devices randomly distributed across the grid should not appreciably affect the overall performance. The transmissionline model of the grid is an inductance in series with the diode capacitance and negative conductance. The inductance of the grid tunes out some of the capacitance of the diodes. The negative conductance gives the grid a reflection coefficient with a magnitude greater than unity.

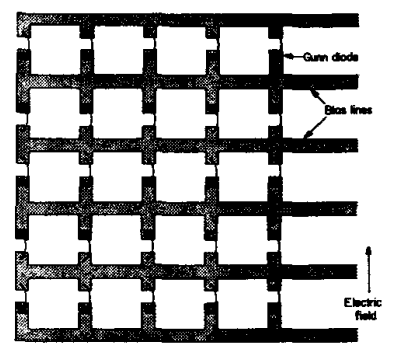

(a)

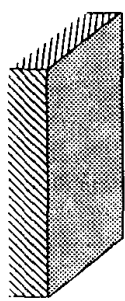

Mirror

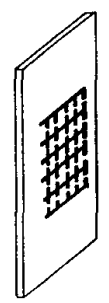

Diode grid

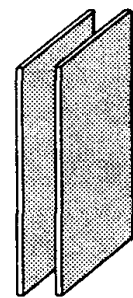

Partiolly tronsparent
reflectior

(b)



(c)

Figure 1. (a) Gunn-diode loaded grid; (b) Power-combiner configuration; (c) Transmission-line equivalent circuit.

The power-combiner configuration is shown on Fig. 1(b). The active grid is placed in parallel with the plates of a Fabry-Perot resonator. One resonator plate is a metal mirror, and the other is a partially transparent reflector which allows some of the power to get out of the cavity. When the diodes are biased in their negative incremental resistance region, they will oscillate with a frequency of a resonator mode. There is no need for an external locking signal, because the diodes couple through the cavity. This configuration can be viewed as a maser, where the active medium is the diode grid. 
The transmission-line model of the entire configuration is given on Fig. 1(c). The mirror appears as a short, and the partially transparent reflector is modeled as a section of transmission line of characteristic impedance $\eta_{d}=377 \Omega / \sqrt{\epsilon_{r}}$. This reflector can be made as a pair of dielectric slabs of a dielectric constant $\epsilon_{r}$ and a quarter-dielectricwavelength thick (we use Stycast slabs with $\epsilon_{r}=12$ ). From the transmission-line point of view, it acts as a matching circuit for the grid impedance, and is analogous to slug tuning in a coaxial line. In this way a wide practical impedance tuning range can be obtained. In principle, loads with a standing-wave ratio up to $\epsilon_{r}^{2}$ can be matched.

\section{GUNN-DIODE GRID AT $10 \mathrm{GHz}$}

Preliminary measurements were done on a 9 -diode grid on a silicon substrate. The devices were found to lock to a single frequency between 10 and $13 \mathrm{GHz}$, depending on the bias and the position of the partially transparent reflector. The power was low $(100 \mu W)$. We believe that one of the reasons was that the design is based on an infinite grid, and our 3 by 3 grid was only a third of a free-space wavelength across. We found that the total power was proportional to the square of the number of diodes. The polarization of the electric field was linear. The far-field $\mathrm{H}$-plane pattern had a single lobe with half-power points at $\pm 20^{\circ}$. The E-plane pattern was two-lobed. Heat-sinking was a problem in this configuration; each diode dissipated $2 \mathrm{~W}$. After 2 minutes of continuous operation, the devices got very hot and their power dropped.

A 100-diode grid is now under construction (Fig. 2). The grid is deposited on a quarter-dielectric-wavelength thick silicon substrate which is held by vacuum to a watercooled copper block. The copper block acts as both a heat-sink and one of the resonator reflectors.

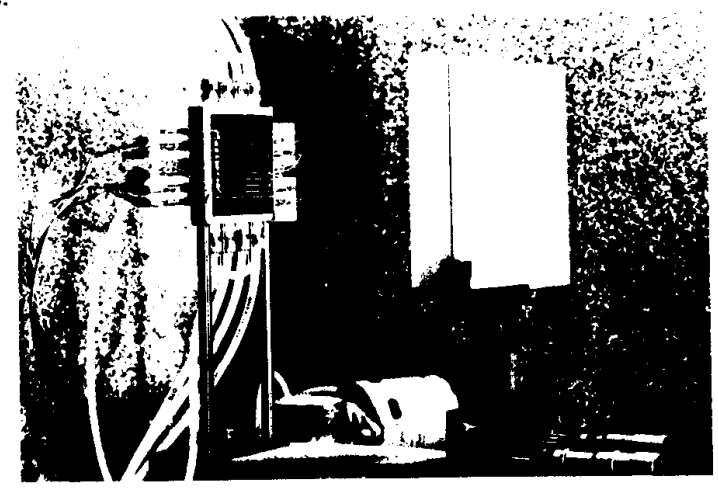

Figure 2. Power-combining measurement setup for a 10 by 10 active grid. 


\section{ACKNOWLEDGMENTS}

This research was supported by the Army Research Office and the Jet Propulsion Laboratory.

\section{REFERENCES}

[1] V. P. Kesan, D. P. Neikirk, T. D. Linton, P. A. Blakey, B. G. Streetman, "Influence of the Transit Time Effects on the Optimum Design and Maximum Oscillation Frequency of Quantum Well Oscillators," 12th International Conference on Infrared and Millimeter Waves, Orlando, Florida, December 1987.

[2] J. W. Mink, "Quasi-Optical Power Combining of Solid-State Millimeter-Wave Sources," IEEE Transactions on Microwave Theory and Techniques, Vol. MTT-34, Pp. 273-279, February 1986 .

[3] K. D. Stephan, "Inter-Injection-Locked Oscillators for Power Combining and Phased Arrays," IEEE Transactions on Microwave Theory and Techniques, Vol. MTT-34, PP. 1017-1025, October 1986.

[4] R. J. Dinger, D. J. White, D. R. Bowling, " $10 \mathrm{GHz}$ Space Power Combiner with Parasitic Injection Locking," Electronics Letters, Vol. 23, pp. 397-398, April 1987.

[5] W. W. Lam, C. F. Jou, H. Z. Chen, K. S. Stolt, N. S. Luhmann, D. B. Rutledge, "MillimeterWave Diode-Grid Phase-Shifters," To be published in the IEEE Transactions on Microwave Theory and Techniques.

[6] C. F. Jou, W. W. Lam, H. Z. Chen, K. S. Stolt, N. C. Luhman, D. B. Rutledge, "MillimeterWave Diode-Grid Frequency Doubler," Submitted for publication in the IEEE Transactions on Microwave Theory and Techniques, October 1987. 